\title{
Écologie politique des paysans alternatifs de l'Empordà (Catalogne) : s'engager entre mer et montagne
}

The Political Ecology of Alternative, Small-Scale Farmers in Empordà

(Catalonia): Working Between the Sea and the Mountain

\section{Sabrina Doyon}

\section{(2) OpenEdition}

Journals

\section{Édition électronique}

URL : http://journals.openedition.org/developpementdurable/16601

DOI : 10.4000/developpementdurable.16601

ISSN : 1772-9971

Éditeur

Association DD\&T

\section{Référence électronique}

Sabrina Doyon, «Écologie politique des paysans alternatifs de l'Empordà (Catalogne) : s'engager entre mer et montagne ", Développement durable et territoires [En ligne], Vol. 11, $\mathrm{n}^{\circ} 1$ | Avril 2020, mis en ligne le 30 avril 2020, consulté le 15 mai 2020. URL : http://journals.openedition.org/

developpementdurable/16601; DOI : https://doi.org/10.4000/developpementdurable.16601

Ce document a été généré automatiquement le 15 mai 2020.

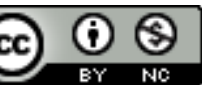

Développement Durable et Territoires est mis à disposition selon les termes de la licence Creative Commons Attribution - Pas d'Utilisation Commerciale 4.0 International. 


\title{
Écologie politique des paysans alternatifs de l'Empordà (Catalogne) : s'engager entre mer et montagne
}

\author{
The Political Ecology of Alternative, Small-Scale Farmers in Empordà \\ (Catalonia): Working Between the Sea and the Mountain
}

Sabrina Doyon

1 La région de l'Empordà, dans le nord de la Catalogne espagnole, est surtout connue pour son esthétique, mise en scène par l'œuvre d'un artiste de la région - Dali - et son tourisme de plage. Depuis une vingtaine d'années, elle se démarque aussi par l'émergence d'une paysannerie alternative à l'agriculture conventionnelle. Celle-ci conjugue des démarches d'écologisation à un processus de territorialisation du système alimentaire. C'est ce que cet article s'attachera à examiner chez des producteurs vitivinicoles et oléicoles ainsi que, dans une moindre mesure, des maraîchers et des éleveurs. Comment sont-ils parvenus à amalgamer ces deux processus? Quelles sont les valeurs qui les guident et les pratiques qu'ils mettent en action? Quelles sont les répercussions sociales et économiques de ces initiatives?

2 Nous proposons d'explorer ces questions grâce à l'approche de la political ecology (Peet et al., 2011; Gauthier et Benjaminsen, 2012) qui étudie les changements environnementaux. Cette perspective critique considère que ces changements ne sont pas neutres, étant plutôt induits par différents mécanismes, notamment des conflits environnementaux, des initiatives de conservation, des perceptions différenciées des problèmes environnementaux et l'émergence "d'identités environnementales" (Robbins, 2004). Afin de comprendre ces mécanismes, la political ecology propose d'analyser l'histoire environnementale locale, les activités et les luttes pour l'accès et le contrôle des ressources et des capitaux, les négociations et les tensions autour de la construction des discours et des récits environnementaux, et l'influence des changements environnementaux sur les inégalités socio-économiques et les processus 
politiques. C'est dans cette perspective que nous examinerons les changements environnementaux récents ayant marqué l'Empordà, et qui s'ancrent dans un contexte socio-économique et politique constituant le socle sur lequel se fonde l'émergence de ces paysans alternatifs.

3 Les recherches portant sur la nouvelle ruralité (Ploeg, 2009) présentent les nouveaux paysans comme souhaitant adapter leurs pratiques à leur milieu naturel, êtres résistants et autonomes à l'égard des modèles conventionnels de production par le truchement de systèmes alimentaires locaux, du développement d'une forte conscience environnementale, de la coopération, l'innovation ainsi que l'engagement social et communautaire (Trauger, 2014; Ribas et al., 2016; Calvário, 2017). Ces études examinent les initiatives de production alimentaire locale et engagée dans des territoires précis (Whatmore et al., 2003 ; Mundler et Laughrea, 2016).

Deux dimensions importantes du rapport à la nature que mettent en œuvre ces paysans ont pu être dégagées. D'une part, ils s'éloignent du rapport conventionnel occidental à la nature (Richardson, 2008) pour s'engager dans une autre « ontologie » (Doyon, 2017 ; Foyer, 2018) où, par des pratiques conscientes et une organisation socio-économique conséquente, la conservation et la protection de la nature sont visées (Veteto et Lokyer, 2008 ; Mormont, 2009 ; Calvário et Kallis, 2017). D’autre part, ils valorisent le paysage et le terroir (Barham, 2003 ; Bérard et Marchenay, 2006) par le truchement de pratiques de production, de savoirs locaux, de référence à l'identité régionale et de réseaux de commercialisation favorisant un ancrage territorial des activités de production (Barham, 2002 ; Ramírez-García et al., 2016). Notre article s'inscrit dans la foulée de ces recherches. Il pose les pratiques et les discours des nouveaux paysans, que nous nommons paysans alternatifs, au cœur de la convergence de l'écologisation et de la territorialisation de l'agriculture, contribuant à la création d'un nouveau paysage régional, mais dont la finalité pourrait leur échapper au profit des producteurs conventionnels.

Les données sur lesquelles repose ce texte sont tirées d'une recherche ethnographique en cours $^{1}$ dans la région de l'Empordà. Cette région compte 68 communes et 140000 personnes habitent ses $1357 \mathrm{~km}^{2}$. Le paysage de l'Empordà est marqué dans la partie montagneuse par une géographie escarpée, des sols granitiques et d'ardoise et une végétation sèche. Dans la plaine, les sols plus fertiles ont été en bonne partie gagnés sur d'anciennes zones inondables littorales et fluviatiles. Balayée par la tramontane, la région surplombe la Méditerranée, qui peut être vue de presque tous les villages. La région a traditionnellement été majoritairement investie par la culture de plantes ligneuses pérennes résistantes aux stress hydriques: chêne-liège, vigne et oliviers en terrasses sèches (pedras secas). La production alimentaire primaire est complétée par des pêches côtières et des élevages de bovins et d'ovins pour la production de viande et de fromages respectivement. Trois parcs naturels s'inscrivent aux marges des terres les plus intensément mises en production: Aiguamolls, qui protège ce qu'il reste de marais de la plaine, Cap de Creus, marquant dramatiquement la plongée des Pyrénées dans la mer Méditerranée, et l'Albera, une aire de cols historiquement célèbres dans l'arrière-pays pyrénéen, frontalière avec la France. Des animaux paissent dans les trois parcs et trois producteurs de vin sont implantés dans les limites du parc du Cap de Creus, d'autres sont dans le parc de l'Albera. Les parcs de la région permettent aussi une apiculture distinctive produisant un «miel de parc écologique ». L'Empordà est enfin marquée par une affluence touristique intense, 
décuplant sa population en période estivale. La très grande majorité des paysans alternatifs produisent en culture bio, certains en biodynamie, et valorisent les espèces locales et ancestrales, de semences, de cépages, d'essences d'arbres à fruits et de races animales. Leur production est fondée sur de petites superficies : en moyenne 5 à 10 ha, rarement plus de 20 ha (une quarantaine pour les producteurs d'olives). Ces producteurs représentent $5 \%$ des 1700 agriculteurs que compte la région. L'analyse révèle qu'ils remettent en question l'histoire environnementale récente de leur région et les imaginaires institués qu'elle porte pour proposer un modèle qui leur est propre, mais non sans ambiguïté. En ce sens, ils mettent en lumière les tensions et les paradoxes propres à ce monde « alternatif et écologique ».

\section{Contexte}

6 L'histoire environnementale récente de la région est marquée par quatre évènements majeurs : 1) la disparition de 220000 ha de vignes par le phylloxera à la fin du XIX ${ }^{\mathrm{e}}$ siècle ; 2) le gel de l'hiver 1956 ayant mené à l'abattage de $90 \%$ des oliviers ; 3) la destruction progressive des écosystèmes côtiers par le développement immobilier à partir des années 1960, et 4) la sécheresse et les feux qui s'intensifient depuis les années 1990. Comme le propose la political ecology, l'histoire environnementale permet de comprendre les changements environnementaux et plus particulièrement les mécanismes menant aux luttes d'accès au territoire et aux luttes idéologiques pour contrôler les récits environnementaux. C'est ce que nous examinerons dans ce qui suit afin de saisir ce qui constitue la base ayant favorisé le démarrage des activités des paysans alternatifs.

7 La faible qualité des sols et la rigueur climatique de la région de l'Empordà semblent en avoir fait, depuis l'époque romaine, un lieu peu prisé pour l'agriculture. Elle est encore dominée par la culture de la vigne. Elle a cependant été durement affectée par la disparition de centaines de milliers d'hectares de vigne dans la décennie 1880, contraignant une vaste proportion de sa population à l'exode (Saguer, 1987, 1989; Pintó, 2008). Ce dépeuplement a forcé de grands propriétaires terriens à instaurer un système plus avantageux pour les paysans afin de maintenir la force de travail dans les champs et d'y travailler les nouveaux hectares de vignes aux pieds américains. Ces grands propriétaires ont également contribué au développement de coopératives de production vitivinicoles et oléicoles pour moderniser et industrialiser la production tout en mutualisant les coûts (Cardaba i Carrascal et Musquera i Felip, 2007 ; Cervantes, 2008 ; Bassegoda Pineda et Puig Vayreda, 2012). La vigne demeure une culture phare dans la région, mais on n'y compte aujourd'hui que 2200 hectares, soit 100 fois moins qu'avant le phylloxera.

8 Lorsqu'est survenu le gel de 1956, la région était marquée par les conséquences socioéconomiques de la diminution majeure de la production viticole, de l'exode et de la guerre civile. Ce gel fut caractérisé par une période de froid intense qui s'est maintenue pendant plusieurs semaines, affectant gravement les oliviers, dont la production était jusque-là toujours bien vivante dans la région (Hosta et Serna, 2005). Avec certains soins et un peu de temps, ces oliviers auraient pu survivre au gel, les quelques spécimens centenaires observables encore aujourd'hui le prouvent. Cependant, la quasi-totalité des arbres fut arrachée par des travailleurs forestiers venus de la Garotxa et des Pyrénées, dont plusieurs se sont sédentarisés dans la région $\left(\mathrm{cp}^{2}\right.$ oléiculteur 
février 2018). En effet, l'Espagne tentait alors de calquer les politiques d'investissement agricole européennes de l'après-guerre et opta pour les mêmes cultures que celles mises en avant à cette époque. La dictature a ainsi soutenu l'arrachage des oliviers, et plus tard de vignes, au profit de la culture du soja, notamment ${ }^{3}$. Le paysage de la région fut profondément transformé par ce changement de culture, à l'instar des pratiques agricoles et des rapports socio-environnementaux en général. Les quelques producteurs d'olives qui survécurent à ces transformations se sont lancés dans la production du cultivar arbequina, une variété andalouse, car il leur était devenu impossible de s'approvisionner en variétés autochtones (argudell, corivell) dans les pépinières régionales, d'où elles avaient disparu (cp oléiculteur janvier 2018).

Trois décennies plus tard, lors de l'adhésion de l'Espagne à l'Union européenne, ces transformations agricoles ont été maintenues et appuyées par les aides qui ont été offertes pour arracher les vignes et les oliviers, visant à conserver l'équilibre de l'offre de produits au sein de l'Union ${ }^{4}$ (Ribas et Sauri i Pujol, 2002 ; Salamana, cp 2018). La très grande majorité des producteurs vitivinicoles et oléicoles (environ 500), toujours en activité dans la région, sont aujourd'hui regroupés dans l'une des trois coopératives, leur âge moyen est d'environ 65 ans. Une cinquantaine, parmi lesquels se trouvent les paysans alternatifs, mais aussi les trois grands domaines régionaux, sont indépendants. Historiquement, les grands propriétaires terriens de la région ont pu s'approprier des terres par dépossession de communaux ${ }^{5}$ (Serrano 2016) et d'autres ont pu les acquérir à la suite de leurs aventures commerciales fructueuses dans les Amériques ${ }^{6}$. Aujourd'hui, l'accès à la terre est ardu en raison de son prix prohibitif, résultat notamment de la spéculation foncière et immobilière ayant culminé dans les années 2000. Depuis moins d'une dizaine d'années, les prix sont demeurés très élevés, mais la demande a changé la donne. D'un côté les vieux agriculteurs du monde coopératif préfèrent garder leurs terres, même si cela implique qu'elle soit improductive et envahie par la garrigue, plutôt que de la vendre à un prix plus bas que celui des transactions en cours avant la crise économique (cp viticulteur février 2018). D'un autre côté, les nouveaux producteurs et les paysans alternatifs qui souhaitent, toujours plus nombreux, se lancer en agriculture ne peuvent acheter de terres et s'établir dans la région en raison des prix trop élevés tant des terres que des logements, gonflés par la demande touristique (Carbonell, 2017). Par ailleurs, les grands domaines et propriétaires terriens de la région ne souhaitent plus acheter ces terres, comme ils l'ont fait jusque dans les années 2000 , préférant d'une part contractualiser la force de travail et l'achat de produits en externalisant les risques, et d'autre part ne pas investir dans des terres agricoles qui pourraient être affectées par les changements climatiques et ne plus permettre une production de vignes intéressante (Biel, 2018 ; Savé, 2018).

10 L'économie agricole de la région a ainsi traversé des périodes difficiles et le tourisme de masse, accompagné par un important développement immobilier, a permis à la région de sortir la tête de l'eau dans les années 1960 grâce au nouveau produit commercial fraîchement nommé «la Costa brava " (Barbaza, 1988). Ce développement a permis à des milliers de propriétaires de vendre des terrains ${ }^{7}$ et de construire des appartements et à encore plus de travailleurs de s'intégrer à l'industrie de la construction. Les cultures de la vigne et de l'olive furent petit à petit abandonnées, leur calendrier étant notamment peu compatible avec celui de la saison touristique, au profit de cette nouvelle économie de service au rendement financier incommensurable vis-à-vis de 
celui de l'agriculture (Barbaza, 1988 ; Compte Freixanet, 2016). De très nombreuses parcelles agricoles ont dans la foulée été abandonnées et reconquises par la garrigue.

11 Ce développement touristique a contribué à la destruction des écosystèmes côtiers fragiles de l'Empordà et il exerce une pression croissante sur les aquifères, dont l'eau est nécessaire pour approvisionner les centaines de milliers de visiteurs estivaux. Cette situation contribue à exacerber les conséquences des épisodes de sécheresse que subit la région depuis les années 1990, dont les derniers furent très préoccupants (Pavon et al., 2002 ; Pavon, 2006 ; Saurí et al., 2010 ; Villares et Roca, 2018). S'ajoutant aux demandes en eau pour l'irrigation des cultures de grains et les centaines d'élevages porcins (375 000 têtes) (Iaeden, 2018), la région se trouve dans une situation précaire quant à son approvisionnement en eau. À ces sécheresses s'ajoutent des feux estivaux destructeurs (Gordi i Serrat, 2009), rasant les terres non cultivées envahies par les arbustaies très combustibles de la garrigue et menaçant la sécurité des établissements humains. Un problème d'eaux usées complète ce portrait, car les municipalités de la région n'ont pas été construites pour traiter les résidus d'un si grand nombre de citoyens en période d'affluence touristique et encore moins les purins de centaines de fermes (Iaeden, 2018).

Les pressions environnementales destructrices ont favorisé l'émergence de groupes environnementalistes dans les années 1970, militant pour l'arrêt du développement immobilier en zone côtière et la protection de ses derniers écosystèmes intacts (Marti, 2017). C'est ainsi qu'ils ont contribué à la création de la première aire protégée, les Aiguamolls, en 1977, appuyés par le premier gouvernement de la région autonome de Catalogne. Les deux autres parcs de la région seront constitués dans le même esprit ${ }^{8}$. Ces groupes environnementalistes font aujourd'hui alliance avec les nouveaux paysans contre le développement d'un macro-camping, contre la prolifération anarchique des porcheries, contre les activités d'une base militaire dans la région viticole et contre le développement d'autoroutes et d'infrastructures énergétiques industrielles (lignes de très haute tension).

Les conséquences de cette histoire socio-environnementale sont diverses, tant en ce qui concerne l'organisation socio-politique que la marginalisation et la différenciation régionale, rendant notamment l'accès à la terre très difficile pour les nouveaux paysans. Avoir marginalisé la production agricole et limité son accessibilité, dilapidé le patrimoine naturel des vignes et des oliviers autochtones et valorisé une agriculture conventionnelle régie par les politiques internationales, dégradé des terres arables, détruit la biodiversité, atrophié la diversité sociale et érodé la vie communautaire des villages, dont le paysage est altéré par de nouvelles banlieues cimentées d'appartements pour touristes aux volets de plastique blancs fermés 10 mois par année, fait partie de ces conséquences.

\section{Les « paysans alternatifs » : qui sont-ils ? Que font- ils?}

14 Cette histoire socio-environnementale est au cœur de l'imaginaire des habitants de l'Empordà et constitue le cadre à partir duquel les paysans alternatifs ont développé leurs pratiques depuis environ 15 ans. C'est un espace où l'accès à la terre est difficile à négocier, où presque toute la production est conventionnelle, bien que les grands vignobles et les coopératives aient entamé depuis trois ans les démarches pour mettre 
quelques hectares en production biologique, où les politiques alimentaires internationales ont un impact local concret sur le paysage et où le développement du tourisme de masse est encore protégé par un système juridique et des politiques municipales, poursuivant le processus de dégradation environnementale.

Les paysans alternatifs proposent des rapports socio-environnementaux différents. Ayant démarré leurs activités dans les années 2000, pour plusieurs d'entre eux, en plein cœur de la crise de 2008 , ils sont très politisés et leurs discours sont ancrés dans ceux de l'alternative écologique et économique globale. Ils sont en relation avec de nombreuses initiatives internationales, la majorité est autodidacte et a complété ses apprentissages par quelques formations ponctuelles. Ils favorisent l'occupation du territoire dans sa complexité et sa diversité, une production alimentaire locale pour une consommation locale, un engagement communautaire et, surtout, un engagement environnemental et écologique. Une sélection de portraits de ces paysans alternatifs, représentatifs de la diversité des profils, des parcours, des types de production menés et des enjeux rencontrés, illustre ces propositions.

Sandra aura bientôt 50 ans. Elle est architecte et a travaillé dans ce domaine pendant 20 ans avant de reprendre la terre de ses beaux-parents pour y produire du vin «nu », "naturel », un produit encore plus bio que le vin biologique, car en plus d'une culture sans intrants, le vin ne contient aucun additif. Sur les 7 hectares de vignes en culture, Sandra parvient à produire 4000 bouteilles par an, ce qui constitue une très petite production. Les terres qu'elle possède avec son conjoint, pompier, sont cernées par un champ de tir de l'armée espagnole et, alors qu'elle désherbe ses vignes, elle enregistre le son produit par les exercices militaires avec son téléphone afin de documenter cette situation qu'elle juge inacceptable. Elle transmet ces enregistrements à l'organisation environnementaliste Iaeden afin de constituer un dossier pour dénoncer cet état de fait. Entre les vignes cultivées avec la «technique en vase », on peut parfois apercevoir des tortues méditerranéennes (Testudo hermanni hermanni), espèce en voie d'extinction, et qui est en repeuplement sur ses terres, grâce à une collaboration avec le Centre de reproduction des tortues de l'Albera. Sandra peut accueillir ces reptiles et en favoriser la reproduction, car elle ne retourne pas le sol dans sa pratique agricole, ce qui protège les œufs enfouis. Quant aux adultes, leurs allées et venues sur sa propriété et les environs sont révélées par des émetteurs GPS. Lorsque Sandra dit: "Je veux pouvoir vivre dignement et sainement de ma production ", on comprend que l'enrichissement personnel n'est pas son objectif premier. Elle veut maintenir et déployer une production écologique sans recourir à des produits de synthèse. Elle souhaite rester à l'écart des mécanismes de marchandisation, de commercialisation et de réglementation qui dominent dans sa région et qui s'établissent notamment par la dénomination d'origine $d u$ vin de l'Empordà. Elle participe néanmoins à certains concours œnologiques qui lui permettent d'avoir une plus grande visibilité, car les habitants de son village et de la région immédiate n'achètent pas ses produits.

Nuria et Jordi, jeunes trentenaires, sont de la région de Barcelone. Ils ont étudié respectivement en communication et en graphisme. Ils ont voulu quitter la ville pour s'investir dans le travail des champs au début des années 2000. Ils ont choisi de s'installer dans l'Alt Empordà en y louant une terre agricole pourvue d'un vignoble. Après quelques années, ils ont fait une offre d'achat au propriétaire. Ce dernier, un homme sans descendance ou famille proche a décidé de leur vendre ses terres. "Nous sommes chanceux, car c'est extrêmement rare qu'un agriculteur accepte de vendre ses terres à 
des jeunes comme nous, d'autant que nous ne sommes pas de la région! " La majorité de leurs vignes sont en production bio, et ils envisagent la biodynamie. Ils ont amélioré leur agro-écosystème en intégrant progressivement des animaux. D'abord des poulets (qui consomment notamment la peau des raisins après leur fermentation et procurent un fumier), puis, plus récemment, des brebis, qui, en hiver, nettoient leurs vignes et leurs vergers d'oliviers et permettent une petite production de fromage. Enfin, ils ont aussi désormais des abeilles, grâce aux conseils et à l'aide d'un ami de Barcelone qui s'y connaît en apiculture.

Erica est l'une des trois grands propriétaires terriens du parc de l'Albera. Biologiste marine de formation, elle a longtemps travaillé en Afrique de l'Est et a repris la ferme familiale à son frère qui voulait tout quitter. Elle y élève des vaches qui ont une dénomination d'origine. "Mes animaux peuvent aller partout, dans la haute montagne et dans la plaine, et ce en toutes saisons! De plus, ils peuvent manger les branches et les feuillages. "Cette race, la vaca de l'Albera, est considérée peu productive, mais donnant une viande de grande qualité recherchée par les restaurateurs prestigieux de la région. Erica participe au plan de conservation des milieux humides et des prés de montagnes, pour lequel elle a été contactée par une organisation environnementaliste qui vise leur réhabilitation.

Cristina produit avec son mari une huile d'olive à partir des 40 hectares d'oliviers qu'elle exploite. Sa trajectoire s'inscrit dans une longue lignée de producteurs d'huile et elle conserve le souvenir des pratiques de ses parents et de ses grands-parents ayant traversé les vicissitudes de la guerre civile et du franquisme. Elle a choisi de produire une huile de qualité et elle est l'une des quatre productrices membres de la dénomination d'origine de l'huile de l'Empordà. Elle n'emploie pas d'herbicides et maintient un couvert végétal au sol. Elle s'oppose cependant aux nouvelles productions bio, car elle dit qu'elles "dénaturent ce qu'est l'olivier et sa culture en Méditerranée » en créant une monoculture intensive qui fragilise les arbres et surexploite les ressources hydriques par l'irrigation. Elle dit que le coût environnemental de la production bio d'olives est trop élevé. Elle définit sa production et son produit comme le fruit de pratiques naturelles conscientes des écosystèmes qui favorisent la biodiversité et elle contribue à maintenir le paysage végétal et le patrimoine naturel de sa région en cultivant et réhabilitant les oliviers centenaires qui sont maintenant une condition de l'attribution du sceau de la dénomination d'origine. Elle transmet ces connaissances et veut éduquer les Catalans à l'importance de cette culture par divers ateliers et visites agrotouristiques.

\section{3. Écologisation des pratiques et des discours}

20 Un des axes majeurs qui traverse les cas ethnographiés est d'abord celui de l'écologisation. On observe de la part des paysans alternatifs un engagement ferme pour la protection de la nature, non pas dans une logique d'exclusion et de séparation entre les humains et l'environnement, où ce dernier serait maintenu sous cloche, mais dans une logique de collaboration entre les deux. Leurs pratiques contribuent réellement au maintien, à la restauration et à la bonification de la biodiversité des agroécosystèmes et des milieux habités. Entre autres pratiques, ils éliminent les additifs, réintroduisent des animaux, favorisent le recyclage sur la ferme et évitent de brûler les résidus de tailles des vignes et des oliviers pour en faire des copeaux, 
contrairement aux producteurs conventionnels. Ils combinent au quotidien des pratiques traditionnelles et modernes (dont les techniques "fils et vase " pour la conduite des vignes et la vinification en amphores de terre cuite et en cuves d'inox) et ils favorisent désormais la réintroduction d'espèces autochtones valorisées pour leur grande résistance, notamment au vent.

21 Ces pratiques favorisent une densité et une diversité d'espèces, dont une grande majorité est bénéfique au rendement de la production : nichoirs pour les oiseaux, abris pour les tortues, étangs pour les batraciens, maintien d'une diversité florale propice aux insectes pollinisateurs, composantes arborées diverses, etc. Leurs pratiques contribuent à ramener du carbone dans les sols, évitent leur compaction, érosion et dégradation. Toutes ces actions concrétisent une écologisation des pratiques, bien qu'elles demeurent minoritaires dans le paysage.

Certains producteurs sont en culture biodynamique, d'autres biologiques, et d'autres encore en transition vers le biologique. L'évidence de la culture en régie minimalement biologique est claire pour les producteurs maraîchers et viticoles alternatifs. En ce qui concerne la production d'olives, la question semble cependant plus nuancée, même débattue par certains. En effet, plusieurs rapportent d'une part que la lutte contre les nuisibles est extrêmement difficile, voire impossible, en raison du grand nombre d'oliviers sauvages dispersés sur le territoire (abandonnés dans la foulée des politiques agricoles européennes), contribuant à garder actives et à propager les maladies de l'olivier. D'autre part, la majorité désapprouve la culture biologique des oliviers en raison de la «violence » qu'elle implique pour l'arbre. Les oliviers en culture biologique sont selon eux fragilisés, ils sont conduits sur des fils de fer, nécessitent une irrigation constante et doivent être protégés des vents, notamment. Pour la majorité des paysans, ces conditions de culture sont "contre nature» et l'écologisation des pratiques doit d'abord prendre en compte l'intégrité physique « naturelle » de l'olivier, tout en évitant le plus possible d'utiliser les intrants. D'ailleurs, les producteurs alternatifs réprouvent l'utilisation d'herbicides pour nettoyer le sol où poussent les oliviers, une pratique qu'ils attribuent à une habitude esthétique culturelle valorisant un champ " propre », et soulignent qu'un olivier en bonne santé et bien entretenu aura un système racinaire qui ne pâtira pas d'une compétition avec les herbes au sol pour l'accès à l'eau.

L'écologisation des pratiques et des discours s'exprime aussi dans la participation des paysans à des projets de conservation environnementale, aux missions des réserves naturelles, aux luttes des mouvements environnementalistes et à l'éducation environnementale par l'agritourisme. Les paysans alternatifs s'engagent dans l'œnotourisme et l'oléitourisme avec l'intention, d'une part, de reconnecter les habitants de la région avec leur histoire environnementale et agricole afin qu'ils prennent conscience de la richesse de leur terroir, qu'ils s'intéressent aux dynamiques locales, qu'ils valorisent les produits locaux alternatifs et éventuellement soient solidaires des conditions de vie des agriculteurs. D'autre part, ils croient que ce type d'agrotourisme avec des visiteurs étrangers pourra contribuer au développement d'un tourisme plus respectueux et plus impliqué régionalement, s'écartant du tourisme de plage de type « low cost ».

24 Récemment, les paysans alternatifs participent à la conservation environnementale par le biais du programme de protection de tortues ainsi que par la restauration de prairies sauvages des régions montagneuses (els prats d'en dalt). Ces prés concentrent une grande biodiversité endémique et ils se maintiennent par des pratiques de pâturage 
saisonnier à petite échelle. Leur restauration implique donc de conserver ces pratiques et même de les multiplier pour récupérer ceux qui sont en voie d'être envahis par la garrigue. Cette participation a permis, par effet boule de neige, d'entraîner d'autres producteurs, ceux-ci conventionnels, à participer à ces initiatives de conservation en les motivant à faire pâturer ces espaces qui étaient en voie d'être laissés à l'abandon.

Cette mise en valeur de la conservation et de l'écologisation passe aussi par les liens établis avec les trois parcs naturels de la région. Les parcs autorisent en leur sein les activités régionales traditionnelles, telles que le maraîchage, l'élevage, les cultures de la vigne et de l'olivier. Les producteurs qui y développent ces activités utilisent le sceau du parc naturel pour donner une valeur ajoutée à leurs produits. C'est le cas des paysans alternatifs, et aussi plus récemment des coopératives et des grands domaines de production qui possèdent également des hectares de vignes au sein des parcs. La production agricole dans les parcs n'est pas tenue d'être biologique et les producteurs conventionnels jouent, dans le cadre de leur mise en marché, sur l'ambiguïté qui peut subsister entre production biologique et produit de parc naturel. Leur popularité étant croissante, les producteurs conventionnels tentent aussi d'en profiter par le nouveau label de parc naturel.

\section{Territorialisation : éthique et développement local}

Pour les paysans alternatifs de l'Empordà, l'écologisation des pratiques et des discours se cheville étroitement au territoire, à une agriculture qui vient de quelque part (Hinrichs, 2003), en valorisant notamment une éthique du local, une vie sociale, économique et communautaire intégrée dans ces lieux par des activités durables et une vision paysagère de l'usage de l'environnement. La mise en valeur d'un paysage territorial de mar i muntanya, "mer et montagne ", y est le porte-étendard de cette perspective. Les paysans le fondent sur la valeur et la singularité de leurs terres logées entre mer et montagne, donnant un caractère particulier à leurs produits et à leurs activités. La place que joue la tramontane dans cette région est particulièrement valorisée et elle constitue une composante cruciale de ce qui les distingue. La mention de ce paysage revient systématiquement dans tous les entretiens formels et informels que nous avons menés et il est repris par les organisations de dénomination d'origine du vin et de l'huile, notamment, mais non sans débats.

Cette éthique territoriale locale porte également en son centre un intérêt pour des rapports socio-économiques justes et équitables qui seraient indissociables des pratiques écologiques. Les préoccupations pour l'accès à la terre, le développement d'une économie locale plus forte et stable, permettant des salaires et des conditions d'emploi justes et la valorisation de la dignité au détriment de l'enrichissement individuel, sont cruciaux pour ces acteurs. Les prix élevés et le coût de la vie font en sorte que les travailleurs qu'ils emploient ne peuvent habiter sur le territoire, très mal desservi en transport en commun, et qu'ils doivent se déplacer depuis la capitale régionale en voiture. Ils sont aussi critiques de certaines pratiques de producteurs locaux qui, par le système de travail au noir notamment, paieraient mal leurs employés agricoles et les maintiendraient dans une situation précaire.

Ce souci pour des rapports socio-territoriaux plus justes passe également par l'achat et la consommation de produits locaux. Cependant, les paysans alternatifs sont confrontés à une dure réalité : leurs produits sont recherchés par les touristes, promus dans des 
foires visitées par les étrangers et vendus à l'international, mais ils sont boudés par une très grande majorité des habitants de la région. Tant les produits maraîchers que les vins $^{9}$ sont peu consommés localement, dans les maisonnées ou dans les restaurants. Ce paradoxe atterre les paysans. Ils croient que la consommation locale de leurs produits permettrait de tendre vers le développement de la souveraineté alimentaire et une certaine indépendance économique des marchés conventionnels intégrés, comme le sont les productions de porc et de grains.

Leur éthique territoriale s'ancre aussi dans une vision du développement local qui s'écarte du modèle mis en avant depuis les années 1950, marqué par une urbanisation sauvage des côtes, la dépendance à l'économie du tourisme et l'agriculture globalisée. En ce sens, ils s'impliquent notamment dans les mouvements environnementaux qui dénoncent ces développements et ils ont participé en 2017, aux côtés d'agriculteurs conventionnels membres d'une des coopératives de la région, à une tractorada, une manifestation appuyée par les tracteurs des paysans de la région pour dénoncer l'établissement d'un macro-camping au cœur de terres agricoles. Le style de développement de ce type de camping massif, qui relève selon eux d'une autre époque, les a particulièrement choqués, tout comme l'emplacement choisi pour le faire, une zone agricole plus éloignée de la côte qui n'avait pas jusqu'à présent été impactée par le développement urbain et les constructions touristiques. On observe dans cette lutte une convergence de différents types d'acteurs, pas seulement les paysans alternatifs, autour d'une certaine vision de la territorialisation.

Cet intérêt pour la territorialisation par des acteurs conventionnels s'observe aussi dans les processus de mise en place et de fonctionnement des dénominations d'origine (DO) des vins et de l'huile de l'Empordà. Ces DO s'appuient fortement sur le « nouveau » paysage de mer et montagne tant valorisé par les producteurs locaux. Les démarches pour l'établissement des DO ont été réalisées par des producteurs conventionnels de la région avec l'intention de regrouper les producteurs et leur donner une visibilité sur la scène nationale et internationale. La DO implique des processus de normalisation et d'homogénéisation des mécanismes de production. L'adhésion à cette certification est ouverte à tous les producteurs de la région dont les cultivars correspondent à ceux exigés par la DO et qui peuvent en payer les frais annuels.

\section{Mar i muntanya : convergences et frictions d'un nouveau paysage}

31 L'Empordà a été marquée par des évènements environnementaux qui, conjugués à des politiques agricoles et de développement, ont mené à une transformation du paysage, notamment par de nouvelles cultures de grain, l'abandon des vignes et des oliveraies, la plantation de pins dans certaines parcelles, le pullulement des porcheries et le développement immobilier côtier. Ce contexte a entre autres favorisé l'utilisation d'intrants, phytocides et insecticides, dans l'agriculture qui a embrassé les principes de l'industrialisation, du productivisme, de l'intégration et du modernisme, un processus s'inscrivant dans un "régime alimentaire" (food regime) globalisé délétère pour l'environnement, qui déterritorialise la production alimentaire et la vie même des producteurs (Friedman, 2016). Ce processus de détérioration se poursuit désormais de manière aiguë par la contamination des aquifères par les porcheries, le manque de ressources pour le traitement des eaux, des pénuries d'eau potable et une sécheresse 
régionale généralisée qui s'exprime par des feux dévastateurs, que seules les cultures de vignes et d'oliviers restants parviennent à freiner ${ }^{10}$.

Les paysans alternatifs de la région rejettent ces pratiques et les discours qui les soustendent et proposent un modèle combinant une écologisation des pratiques et leur ancrage territorial, visant un mode de vie digne tant pour eux-mêmes que pour la population régionale (Di Masso et al., 2014 ; Di Masso et Zografos, 2015). L'amalgame de ces deux processus, notamment par la revalorisation de pratiques traditionnelles, de cultivars autochtones, d'espèces fauniques et faunistiques endémiques, d'aspects géographiques et climatiques liés aux montagnes et à la côte longtemps dévalorisés et combattus par l'agriculture conventionnelle, contribue à créer le paysage régional de "mer et montagne ». Les paysages, qui ne sont pas des entités fixes, incorporent à la fois des lieux significatifs, des dimensions visuelles et esthétiques, des pratiques, une histoire, une identité et des significations particulières (McIntyre, 1999; Low et Laurence-Zuniga, 2003 ; Nogue et Vicente, 2004 ; Nogué, 2016). Ce paysage de mer et montagne incorpore les différents micropaysages de la région identifiés par l' Observatori del paisatge catalan et donne une portée pragmatique et distinctive à l'Empordà. Cette intégration est importante en liant des lieux, la côte et l'arrière-pays, qui évoluaient relativement parallèlement.

La portée de ce paysage est telle que les autres acteurs de la région, tant du secteur touristique, des services, ou agricole, se l'approprient à leur tour. Des références à ce duo paysager se retrouvent dans les documents et sites de promotion touristique, qui ne valorisent plus uniquement la plage, comme sur les étiquettes des vins, de grands domaines ou de coopératives, dessinées par des artistes locaux. L'appropriation de ce paysage par les producteurs agricoles conventionnels est complétée par celle de l'image des parcs naturels régionaux. Cette juxtaposition favorise une perception de processus d'écologisation des produits et s'inscrit dans une stratégie globale de mise en marché.

La mise en marché par le truchement du paysage est également promue par les dénominations d'origine du vin et de l'huile de l'Empordà. Des recherches ont montré que les mécanismes de certification contribuent généralement à l'écologisation, à la territorialisation et au développement local des régions (Bérard et Marchenay, 2002 ; Urquhart et Acott, 2013; Vázquez et González, 2015 ; Doyon 2017b), en permettant entre autres, comme c'est le cas dans l'Empordà, de valoriser des cultivars autochtones et donc de favoriser une construction du paysage par leur production et reproduction en pépinière. Leurs impacts ne sont cependant pas uniformes (Barham, 2002 ; DeLind, 2011 ; Ribas Serra, 2013 ; Candau et al., 2015). Dans l'Empordà, les DO de l'huile et du vin excluent certains producteurs qui n'ont pas les moyens de payer les certifications, qui sont hésitants à s'engager dans des démarches administratives s'ajoutant à celles déjà exigeantes de la certification biologique, et qui ne souhaitent pas uniformiser et homogénéiser leurs produits pour qu'ils correspondent aux critères de la DO (cépages, cultivars et taux de concentration dans le produit), car ceux-ci leur imposent un goût prédéterminé qui ne reflète pas la diversité, la richesse et la complexité du terroir. Les paysans alternatifs sont aussi critiques du processus de contractualisation des plus grandes maisons et des coopératives membres de la DO qui contractualisent maintenant le travail de petits producteurs devant se conformer à leurs exigences de production et de transformation afin de pouvoir leur vendre leurs produits sans pouvoir exercer de libre choix, l'autonomie étant une valeur fondamentale des paysans alternatifs comme d'autres l'ont aussi souligné (Ploeg, 2009; Narotzky, 2016). Ces 
producteurs sont en marge des mécanismes décisionnels qui impactent la région et son territoire. Des systèmes de production conventionnelle utilisent les discours de la territorialisation pour consolider leurs assises.

Dans ce contexte, des producteurs qui ont traditionnellement été structurellement plus avantagés dans l'organisation économique et politique de la région, comme les grands domaines viticoles ou les coopératives, peuvent-ils s'approprier et instrumentaliser le processus de territorialisation et le dissocier des pratiques d'écologisation qu'avaient développées les paysans alternatifs ? Ces derniers peuvent-ils être mis de côté de ces processus et de ses retombées économiques et politiques au fur et à mesure que les autres prendront une place plus grande grâce aux ressources - financières, administratives et politiques - plus importantes dont ils disposent ?

\section{Conclusion}

Par les pratiques et les discours mis en avant par les paysans alternatifs de l'Empordà, il semble bien que des processus de territorialisation et d'écologisation convergent et se fécondent, contribuant d'une part à favoriser un environnement local durable, ce qui est explicitement au cœur de leur démarche, par des pratiques de conservation et de sensibilisation et un système alimentaire local qui ont des effets visibles et tangibles sur le territoire. D'autre part, leur convergence contribue également à l'introduction du paysage de mer et montagne, un nouveau référent culturel ancré dans des lieux et des pratiques historiques locales englobant la côte et l'arrière-pays de l'Empordà. Ce paysage trouve écho dans la population que nous avons pu enquêter et est repris dans la promotion de différentes activités locales, foires agricoles, tourisme de montagne, écotourisme de pêche côtière et production agricole, notamment.

Les discours de territorialisation, d'écologisation et l'image du paysage de mer et montagne se voient désormais récupérés, dans des géométries variables, par les acteurs conventionnels du monde agricole. Ces acteurs ont émergé historiquement à la faveur d'évènements environnementaux et de politiques de développement nationales et internationales et ils sont ceux qui détiennent le contrôle sur les principales ressources de la région: les terres, les politiques locaux, l'accès aux financements étatique, les patrimoines. Ils ont historiquement emboîté le pas à la mondialisation de la production alimentaire, à l'industrialisation, au productivisme, à la déterritorialisation et à l'utilisation d'intrants chimiques, un modèle que les paysans alternatifs rejettent et veulent transformer. Ces producteurs conventionnels proposent maintenant une lecture de l'écologisation et de la territorialisation qui joue sur les codes, sans fondamentalement changer de modèle de production. Cela peut-il marginaliser les paysans alternatifs émergents? Créer une plus grande différenciation socioéconomique et territoriale? Il sera intéressant d'interroger la façon dont des mécanismes de territorialisation et d'écologisation peuvent être appropriés, dominés ou dévoyés par certains dans un contexte de distribution inégale des ressources environnementales, politiques, économiques et culturelles. 


\section{BIBLIOGRAPHIE}

Barbaza Y., 1988, El paisatge huma de la costa brava, Barcelona, Edicions 62.

Barham E., 2002, « Towards a theory of values-based labeling », Agriculture and Human Values, vol. $19, \mathrm{n}^{\circ} 4$, p. 349-360.

Barham E., 2003, « Translating terroir : the global challenge of French AOC labeling », Journal of Rural Studies, vol. 19, n 1, p. 127-138.

Bassegoda Pineda E., Puig Vayreda E., 2012, « Espolla », Quaderns de la revista de Girona, $\mathrm{n}^{\circ}$ 158, $96 \mathrm{p}$.

Bérard L., Marchenay P., 2002, Un exemple de «non-patrimonialisation » : la carpe en Dombes, Montpellier, Centre national de la recherche scientifique, « Ressources des terroirs - cultures, usages, sociétés ", Études et documentation.

Bérard L., Marchenay P., 2006, « Biodiversité culturelle, productions localisées et indications géographiques », III Congreso internacional de la Red SIAL « Alimentación y Territorios ».

Biel C., 2018, Mesures d'adaptació i mitigació de l'agricultura al canvi climàtic, colloque « Canvi climàtic a la plana Empordànesa », Castelló d'Empúries.

Calvário R., 2017, « Food sovereignty and new peasantries : on repeasantization and counterhegemonic contestations in the Basque territory ", The Journal of Peasant Studies, vol. $44, \mathrm{n}^{\circ} 2$, p. $402-420$.

Calvário R., Kallis G., 2017, « Alternative Food Economies and Transformative Politics in Times of Crisis : Insights from the Basque Country and Greece », Antipode, vol. 49, n³, p. 597-616.

Candau J., Deldrève V., Deuffic P., 2015, « Agriculteurs, pêcheurs et forestiers face à l'impératif environnemental », in Arpin I., Bouleau G., Candau J., Richard-Ferroudji A. (dir.), Activités professionnelles à l'épreuve de l'environnement, Toulouse, Octarès, p. 93-113.

Cardaba i Carrascal M., Musquera i Felip S., 2007, El Celler Cooperatiu d'Espolla. Els inici d'un projecte (1906-1039), Figueres, Brau Edicions.

Cervantes C. R., 2008, « Las Sociedades Agrarias de Transformación en España : un análisis histórico ", CIRIEC-España, Revista de economía Pública, Social y Cooperativa, nº 63, p. 65-87.

Carbonell E., 2017, « Regards sur l'accueil des migrants : modèles et expériences de travail dans l'Alt Empordà et les Pyrénées-Orientales », Escala, Projet ProspecTsaso EFA019/15, 46 p.

Compte Freixanet A., 2016 (1964), El Alto Ampurdan, Figueres, Institut d'estudis Empordànesos.

DeLind L. B., 2011, « Are local food and the local food movement taking us where we want to go ? Or are we hitching our wagons to the wrong stars? ", Agriculture and Human Values, vol. $28, \mathrm{n}^{\circ}$ 2, p. 273-283.

Di Masso M., Rivera-Ferre M. G., Espluga J.-L., 2014, « The Transformative Agrifood Movement in Catalonia : Operational Divergences in the Construction of Food Sovereignty ", Research in Rural Sociology and Development, vol. 21, numéro « Alternative Agrifood Movements : Patterns of Convergence and Divergence », p. 159-181.

Di Masso M., Zografos C., 2015, « Constructing food sovereignty in Catalonia : different narratives for transformative action », Agriculture and human Values, vol. 32, n 2, p. 183-198. 
Doyon S., 2017a, « Are Alternative Farmers Shifting the Paradigm? New Ruralities, Environment, and Conservation in Catalonia », conférence invitée : Distinguished women scholars lecture, université de Victoria.

Doyon S., 2017b, « Mise en marché et certification de l'anguille argentée et de l'esturgeon noir de l'estuaire du Saint-Laurent : des “vendredis maigres" aux produits fins »Canadian Food Studies, vol. $4, \mathrm{n}^{\circ} 1, \mathrm{p} .87-107$.

Friedmann H., 2016, «Food Regime Analysis and Agrarian Questions : Widening the Conversation », Journal of Peasant Studies, vol. 43, n 3, p. 671-692.

Foyer J., 2018, « Syncrétisme des savoirs dans la viticulture biodynamique : incorporation dans l'expérience et le sensible et trajectoire initiatique ", Revue d'anthropologie des connaissances, vol. $12, \mathrm{n}^{\circ} 2$, p. 289-321.

Gautier D., Benjaminsen T. A., 2012, Environnement, discours et pouvoir, Versailles, Quæ.

Gordi i Serrat J., 2009, L'evolucio del paisatge forestal al les terres gironines a la segona meitat del segle $X X$, Girona, Centre de recerca d'historia rural, Universitat de Girona.

Hinrichs C. C., 2003, « The practice and politics of food system localization », Journal of Rural Studies, vol. $19, \mathrm{n}^{\circ} 1$, p. 33-45.

Hosta M., Serna E., 2005 (1956), L'any de la Fred. L'observatori meteorologic de Girona, Figueres, Consell Comarcal de l'Alt Empordà.

Iaeden, 2018, Plataforma Salvem l'Empordà de purins, https://iaeden.cat/ salvemlEmpordàdepurins/, consulté le 11/06/2018.

Low S., Laurence-Zuniga D., 2003, The Anthropology of Space and Place : Locating Culture, Malden, Blackwell Publishing.

Martí Á. (dir.), 2017, dossier spécial « Preservar L'Empordà », Revista Alberes, nº 18, Editorial Gavarres.

McIntyre C., 1999, The Spatial Control of Meaning Through the Mapping and Marketing of Patrimony in Catalonia, Spain, thèse de doctorat, anthropologie, Johns Hopkins University Baltimore, 401 p.

Mormont M., 2009, « Globalisations et écologisations des campagnes », Études rurales, $\mathrm{n}^{\circ} 183$, p. $143-160$.

Mundler P., Laughrea S., 2016, « The contributions of short food supply chains to territorial development : A study of three Quebec territories ", Journal of Rural Studies, vol. 45, p. 218-229.

Narotzky S., 2016, « Between Inequality and Injustice : Dignity as a Motive for Mobilization During the Crisis », History and Anthropology, vol. 27, n 1, p. 74-92.

Nogué J., 2016, « La génesis y la evolución de la valoración moderna del paisaje en Cataluña », Cuadernos Geográficos, vol. 55, n² 2, p. 28-45.

Nogué J., Vicente J., 2004, « Landscape and national identity in Catalonia », Political Geography, vol. 23, p. 113-132.

Pavón D., 2006, « Antecedentes de los grandes proyectos de irrigación en el llano del Alto Ampurdán. La opción fracasada de los grandes regadíos catalanes del siglo XIX », Revista electrónica de geografía y ciencias sociales, vol. XI, n²29, p. 741-798.

Pavón D., Ventura M., Ribas A., Serra P., Saurís D., Breton F., 2002, « Land use change and socioenvironmental conflict in the Alt Empordà county (Catalonia, Spain) », Journal of Arid Environments, vol. 54, $\mathrm{n}^{\circ}$ 3, p. 1-10. 
Peet R., Robbins P., Watts M. J. (dir.), 2011, Global political ecology, Londres, New York, Routledge. Pintó J., 2008, « El paisatge de la vinya. Valors culturals i ambientals », ACE, Revista de Enología, vol. 83, p. 18-23.

PloegJ. D., 2009, The New Peasantries: Struggles for Autonomy and Sustainability in an Era of Empire and Globalization, New York, Earthscan.

Ramírez-García S., Inmaculada Mancha-Cáceres O., Del-Canto-Fresno C., 2016, « Las agriculturas territorializadas, oportunidades y retos frente al paradigma agroindustrial », Documents d'Anàlisi Geogràfica, vol. 62, n 3, p. 639-660.

Ribas A., Sanamaña I., Pavón D., 2016, « Noves Ruralitats. Presentacio », Documents d'Anàlisi Geogràfica ", vol. 62, nº 3, p. 483-485.

Ribas A., Sauri i Pujol D. (dir.), 2002, Canvis Socioambientals a l'Alt Empordà (1950-2000). Natura i historia en l'evolucio recent del paisatge altEmpordànès, Girona, Universitat de Girona.

Ribas Serra J., 2013, Welcome to Verema : Procés de mercantilització de la tradició i el patrimoni. Et cas de la DO Alella, thèse de doctorat, anthropologie sociale, Universitat de Barcelona, Barcelona, $274 \mathrm{p}$.

Richardson M., 2008, Polycultures of the Mind: Organic Farmers in Quebec and the Recovery of Agency, thèse de doctorat, anthropologie, université Laval, Québec, 457 p.

Robbins P., 2004, Political Ecology. A Critical Introduction, Oxford, Blackwell Publishing Ltd.

Saguer E., 1987, « La fil.loxera a les comarques gironines », Congrés Històric Internacional del Centenari de la Fil.loxera i el Cava, nd, Sant Sadurní d'Anoia (España).

Saguer E. (dir), 2005, Els ultims hereus. Historia oral dels proprietaris rurals gironins, 1930-2000, Barcelona, Generalitat de Catalunya Departament de Cultura.

Saurí D., Breton F., Ribas A., Llurdes J. C., Romagosa F., 2010, « Policy And Practice The Ecological Values of Traditional Land Use in Low-lying Coastal Environments : The Example of the Aiguamolls de L'Empordà, Costa Brava1 ", Journal of Environmental Planning and Management, vol. $43, \mathrm{n}^{\circ} 2$, p. 277-290.

Savé R., 2018, «Efectes del canvi climàtic en l'agricultura, Colloque « Canvi climàtic a la plana Empordànesa », 3 février 2018, Castelló d'Empúries.

Serrano L., 2016, Tancar la terra. Practiques de proprietat i dinamiques socials (Catalunya, 1850-1910), Girona, Centre de recerca d'historia rural, Universitat de Girona.

Trauger A., 2014, « Toward a political geography of food sovereignty : transforming territory, exchange and power in the liberal sovereign state », The Journal of Peasant Studies, vol. $41, \mathrm{n}^{\circ} 6$, p. 1131-1152.

Urquhart J., Acott T. G., 2013, « Re-connecting and embedding food in place : Rural development and inshore fisheries in Cornwall, U. K», Journal of Rural Studies, vol. 32, p. 357-364.

Vázquez A. M., González P. A., 2015, « Managing collective symbolic capital through agro-food labelling : Strategies of local communities facing neoliberalism in Spain », Journal of Rural Studies, vol. 41, p. 142-152.

Veteto J. R., Lockyer J., 2008, « Environmental Anthropology Engaging Permaculture : Moving theory and Practice Toward Sustainability », Culture and Agriculture, vol. 30, n 1-2, p. 47-58.

Villares M., Roca E., 2018, La percepció social del canvi climàtic : Els riscos i les amenaces als Aiguamolls de l'Empordà, Colloque « Canvi climàtic a la plana Empordànesa », Castelló d'Empúries. 
Whatmore S., Stassart P., Renting H., 2003, « What's alternative about alternative food networks? », Environment and Planning A, vol. 35, n 3, p. 389-391.

\section{NOTES}

1. Deux cents projets ont été sélectionnés en fonction de leur implication dans la pratique d'une activité d'utilisation et de mise en valeur de la nature misant sur la protection ou une inflexion environnementale, et alternative aux productions conventionnelles depuis 2005, notamment l'agriculture biologique, la pêche durable et l'écotourisme. Une recherche sur internet (notamment sites des municipalités, des organisations de production biologique, des marchés locaux, de la Région, d'organismes environnementalistes et écologistes), un suivi téléphonique puis directement sur le terrain auprès de ces personnes ont permis de consolider cette sélection. Un second corpus de 70 projets a été choisi par la technique de l'échantillonnage non probabiliste visant une représentativité géographique, des secteurs de production, des variations socio-économiques entre les projets, des dates de fondation, des origines des porteurs. Les entrevues ont porté sur leur position face au système économique global et local, les dimensions de leurs rapports à la nature, à la conservation environnementale et à leur région. Des ethnographies des projets, plus particulièrement des entretiens informels, des observations participantes et des observations des lieux, des caractéristiques, des pratiques, des activités et des acteurs ont également été réalisées pendant cette période. Nous avons enfin effectué des recherches dans les fonds d'archives de l'Empordà (archives de l'Alt Empordà, archives des municipalités sélectionnées, archives des coopératives agricoles de la région, archives du groupe environnementaliste régional).

2. Nous référons aux communications personnelles par l'acronyme « $\mathrm{cp}$ ». Ces communications proviennent de paysans alternatifs rencontrés pendant la recherche. Pour respecter la confidentialité des participants à la recherche, les communications personnelles et les extraits d'entrevues sont anonymes dans le texte. Les communications personnelles avec des chercheurs ne le sont pas.

3. Afin d'écouler les surplus d'huile produits par la culture du soja (l'Espagne, contrairement à l'Europe, a acheté les grains entiers, créant un excédent d'huile à écouler après la production du tourteau), l'huile d'olive a alors été faussement accusée par l'État espagnol des pires maux et a recommandé d'en limiter la consommation (Compte Freixanet, 2016 (1964) et Salamana, communication personnelle février 2018).

4. Le nombre d'hectares de vignes et d'oliviers en culture est réglementé et fixé par l'UE. Certains producteurs ont aussi pu recevoir des aides pour reforester les terres mises à nu avec des pins afin de limiter l'érosion (Ribas, $c p$, février 2018).

5. La période de la République a failli mettre un terme à ces patrimoines familiaux, mais avec le régime franquiste, les riches propriétaires qui avaient fui sont revenus réclamer leurs biens, avec compensation. Ces patrimoines ont fait travailler une grande partie de la population pour leur seul bénéfice dans un système que plusieurs qualifient de "féodal moderne ", et engrangé et sécurisé les actifs et les bénéfices économiques en se maintenant à la tête des mairies et des autres instances des pouvoir locaux.

6. Les grands propriétaires fonciers de la région ont, de manière générale mais pas absolue, toujours été près du pouvoir et de l'appareil d'État, leur garantissant des 
positions politiques clés au sein des administrations municipales et régionales (Saguer, cp, février 2018, voir aussi Saguer, 2005).

7. Il est intéressant de noter qu'avant le boom immobilier des années 1960 , les jeunes qui héritaient d'un terrain en bord de mer étaient perçus comme les "perdants » de la famille, car ces terres étaient considérées comme n'étant bonnes à rien. Ironiquement, ce sont eux qui ont fait le plus de profit avec l'avènement du tourisme.

8. Le parc du Cap de Creus a même démoli un centre touristique du Club Med afin de renaturaliser la zone en 1997.

9. Le cas s'applique moins à l'huile, mais sa consommation locale est par ailleurs en diminution selon les producteurs locaux.

10. Les habitants locaux nomment ces cultures des talla-foc, des coupe-feu.

\section{RÉSUMÉS}

La région de l'Empordà, dans le nord de la Catalogne espagnole, voit l'émergence, depuis une vingtaine d'années, d'une paysannerie alternative à l'agriculture conventionnelle conjuguant des démarches d'écologisation à un processus de territorialisation du système alimentaire. C'est ce que cet article s'attachera à examiner chez des producteurs vitivinicoles et oléicoles ainsi que, dans une moindre mesure, des maraîchers et des éleveurs. Comment sont-ils parvenus à amalgamer ces deux processus? Quelles sont les valeurs qui les guident et les pratiques qu'ils mettent en action? Quelles sont les répercussions sociales et économiques de ces initiatives ? À l'aide de l'approche de la political ecology, nous proposons d'explorer les changements environnementaux récents ayant marqué l'Empordà, et qui s'ancrent dans un contexte socioéconomique et politique constituant le socle sur lequel se fondent l'émergence de ces paysans alternatifs ainsi que les pratiques et les discours qu'ils mettent en avant. L'analyse révèle qu'ils proposent un modèle qui leur est propre, conjuguant mer et montagne, mais non sans ambiguïté. En ce sens, ils mettent en lumière les tensions et les paradoxes propres à ce monde « alternatif et écologique ».

The Empordà region in the north of Spanish Catalonia has seen the emergence, in the last 20 years, of small-scale farming which is an alternative to conventional agriculture and which combines an increasing use of ecological practices and a regional approach to food production. The present article examines this type of farming among wine - and olive-sector producers as well as, to a lesser degree, fruit and vegetable producers and livestock breeders. How have they succeeded in combining these two processes? What are the values that guide them in their practices? What are the social and economic repercussions of these initiatives? Using the political ecology approach, we explore the recent environmental changes in Empordà. The socioeconomic and political context in which they have taken root represents the foundation on which small-scale, alternative farmers are basing their practices and their discourse. Our analysis revealed that they have their own unique model which combines the sea and the mountain, albeit somewhat ambiguously. As such, they shed light on the tensions and paradoxes that are inherent in this « alternative, ecological » approach. 
INDEX

Mots-clés : anthropologie, Empordà, Espagne, political ecology, agriculture alternative, agriculture écologique

Keywords : anthropology, Empordà, Spain, political ecology, alternative farming, ecological farming

\section{AUTEUR}

\section{SABRINA DOYON}

Professeur département d'anthropologie, université Laval, Québec, Canada. sabrina.doyon@ant.ulaval.ca 